\title{
ON THE BENEFITS OF A REDUCTION OF MODAL PREDICATES TO MODAL OPERATORS
}

\author{
VOLKER HALBACH \\ University of Oxford
}

For many modal notions English contains a predicate along with an adverb: is necessary is a predicate expression; 'necessarily' is an adverb. ${ }^{1}$ In formal languages this distinction is mirrored by the contrast between modal operators and modal predicates. A modal operator has to be combined with a formula to obtain a new formula; a predicate has to be combined with a singular term. The symbol $\square$ of modal logic is a modal operator; from the syntactical point of view, $\square$ behaves like the negation symbol: written in front of a formula it yields a new formula. The symbol $\square$ is often used to symbolise necessity. Similar remarks apply to many modal notions other than necessity such as analyticity, being known, being believed, being a priori, being true in the future.

In some cases one can easily dispense with the predicate for necessity. The sentence

The proposition that water is $\mathrm{H}_{2} \mathrm{O}$ is necessary.

may be taken to be equivalent to

Necessarily, water is $\mathrm{H}_{2} \mathrm{O}$.

There may be some qualms about the correctness of this paraphrase: The original version seems to be about a certain proposition while the second does not refer to propositions at all; but here I'm not concerned with these

\footnotetext{
${ }^{1}$ Here I am dealing exclusively with adverbial phrases that can be understood as modificators of entire sentences not of parts of sentences. Some of what I am saying applies also to modificators of verbs (or, more generally, of predicates), but here I do not want to go into the intricacies of such cases. Consequently, I focus on de dicto-modalities only.
} 
qualms, as there are far more problematic cases where one does not even have an obvious candidate of the above kind for a paraphrase.

Sentences involving a predicate of necessity and quantification over the objects that may be necessary cannot be easily reformulated without the predicate:

(i) All laws of physics are necessary.

(ii) Some necessary propositions are not a priori.

(iii) All mathematical truths are necessary.

Therefore, disallowing a predicate of necessity and keeping the adverb or the modal operator only, seems to result in a restriction of the expressive power of the language.

If necessity is formalised as the modal operator $\square$, the above sentence (i)-(iii) cannot be formalised without further tricks. The sentence $\forall x(\operatorname{Law}(x) \rightarrow \square(x))$ not well formed. If necessity is treated as a predicate, these sentences can be formalised in a straightforward way.

Despite the problems with quantified sentences and against Quine's advice, philosophical logicians and other philosophers have continued to treat necessity as a modal operator and not as a predicate of certain objects. There might be a socio-historical explanation for this adherence to the operator conception: the paradoxes arising on the predicate account (Montague 1963) and the rise of possible-worlds semantics for modal logic surely contributed to the success of the operator account as elegant technical frameworks for the operator conception became available. Here in this paper I will argue that there are also strong philosophical motives for eliminating predicates of necessity in favour of the corresponding adverbs or operators.

\section{First Benefit: Modal Logic}

If the predicate conception of necessity is adopted, then modal logic is no longer adequate for formal treatments of necessity, and modal logic would have to be rejected as a general framework for studying modal notions. Together with modal logic, presumably also its possible-worlds semantics, at least in its usual form, would have to go. As large parts of philosophy are based on possible worlds as a basic tool (or even more), philosophers should have a strong interest in trying to retain the operator conception of 
necessity as the only conception and not to admit along with the operator conception also the predicate account of necessity. If the operator approach is rejected, then many uses of intensional logics are called into doubt and with it large parts of philosophical logic from deontic over epistemic to temporal logic.

Of course, the existence of a large body of philosophical work based on the adequacy of modal logic and possible-worlds semantics does not show that the operator approach must be sound. But many philosophers would be reluctant to to relinquish all this work.

The existence of possible-worlds semantics for necessity and other modal notions, as developed by Halbach et al. (2003), may be seen as an attempt to salvage some insights gained from modal logic and possibleworlds semantics if one opts for the predicate approach. But as Halbach et al. (2003) demonstrated, the predicate conception is subject to severe constraints because of the paradoxes from diagonalisation, and thus also the possible-worlds semantics for modal predicate deviates in many important aspects from the usual possible-worlds semantics for operators.

\section{SECOND BENEFIT: PARADOXES FROM SELF-REFERENCE}

Montague (1963) used a strengthening of the liar paradox to question predicate approaches to the analysis of necessity. Here is a variation of Montague's paradox with the epistemic-modal predicate 'is known (by someone)'.

(M) The proposition expressed by sentence $M$ is not known.

If the proposition expressed by sentence labelled (M) is known, then, by the $a b$ necesse ad esse-principle or factivity of knowledge, the proposition expressed by sentence $M$ is not known, because this is was the sentence says. Hence the proposition expressed by sentence $M$ is not known, that is, sentence $\mathrm{M}$ is established, and therefore it is known, which is a contradiction.

For the paradox it is not required that 'is known' is conceived as a predicate of propositions. One could equally well use sentences in the place of propositions if one is worried about the ontology of propositions and if one thinks that sentences rather than propositions are the objects that can be known. 
Montague's paradox is a variation or strengthening of the liar paradox (for an analysis and comparison see Halbach et al. 2003), but it applies to many further notions. There are also more sophisticated paradoxes such as Curry's paradox. One important ingredient for these paradoxes is diagonalisation or self-reference. The predicate 'is known' cannot be eliminated and be replaced with a construction that works like an adverb, for instance with the phrase 'as is known'.

Possible-worlds semantics shows that the operator is not threatened by paradox in the same way as the predicate account of modal notions.

\section{THIRD BENEFIT: PARADOXES FROM THE INTERACTION OF MODAL PREDICATES}

Even once the paradoxes from self-reference concerning the different modal notions are resolved in the one or the other way, other paradoxes may well remain that arise from the interaction of two or more modal predicates. Examples of such paradoxes were given by Horsten and Leitgeb (2001), Niebergall (2006), Halbach (2006), Halbach (2008, and Petzolt (2009). Here I provide a simple example. In the example the paradox arises from the interaction of predicates for necessity and aprioricity.

The following axioms and rules are inconsistent with a basic theory of syntax:

A1 If ' $A$ ' is a priori, then $A$ (where $A$ does not contain 'a priori')

A2 rule of inference: If ' $A$ ' has been proved, one may infer " " $A$ " is a priori.' (where $A$ does not contain 'a priori')

A3 If ' $A$ ' is necessary, then $A$ (where $A$ does not contain 'necessary')

A4 rule of inference: If ' $A$ ' has been proved, one may infer " " $A$ " is necessary.' (where $A$ does not contain 'necessary')

If the other axioms and rules don't allow one to prove contingent or a posteriori truths, then A1-A4 ought to be intuitively correct.

Since in the axioms in A1 the predicate of being a priori applies only to sentences without this predicate, it should also be plausible that Montague's paradox does not go through for this theory of a prioricity. In fact, under weak assumptions the consistency of A1 and A2 plus a base theory can easily be established; the same holds for A3 and A4. Basically, in this 
theory Montague's paradox is avoided by invoking Tarski's solution to the liar paradox where the truth predicate does not provably apply to any sentence containing the very same truth predicate.

Using Gödel's diagonalisation technique one can obtain a sentence $D$ with the following property: ${ }^{2}$

$D$ if and only if 'the sentence " $D$ " is necessary' is not a priori.

The sentence can be chosen in such a way that it does not contain the predicate 'is necessary', not even within quotation marks. Mentioned occurrences of expressions, that is, occurrences within quotation marks, can be replaced be using structurally descriptive names (or other names) for expressions in the sense of Tarski 1935 or by using Gödel codes. In order to keep the following discussion more perspicuous I assume that in A1, for instance, $A$ must contain no used occurrence of 'is apriori'; mentioned occurrences can be avoided by using the mentioned techniques. Analogous remarks apply to the other type restrictions in A2-A4 as well.

Thus, if the sentence 'the sentence " $D$ " is necessary' is a priori, then not- $D$. But also if the sentence 'the sentence " $D$ " is necessary' is a priori, then the sentence " $D$ " is necessary by A, and therefore, by A, also $D$. It follows that the sentence 'the sentence " $D$ " is necessary' is not a priori, because the assumption that it is a priori implies both, not- $D$ and $D$.

Therefore $D$ follows, as $D$ is equivalent to the claim that 'the sentence " $D$ " is necessary' is not a priori. Applying A4 to $D$ yields that $D$ is necessary. Using A2 I conclude that 'the sentence " $D$ " is necessary' is a priori. This is a contradiction.

In this derivation the predicate of aprioricity has been applied only to sentences not containing this predicate, and the predicate of necessity has been applied only to sentences not containing the predicate expressing necessity (except for merely eliminable mentioned occurrences). Of course $D$ is 'somehow' about a sentence containing the aprioricity predicate, but this is allowed in solutions of the paradoxes relying on a distinction between an object and metalanguage. Ruling out such indirect occurrences of predicate

\footnotetext{
${ }^{2}$ For obtaining self-reference I cannot invoke the informal trick of labelling a sentence labelled with a number that refers to that very same number. Because then the derivations would depend on the contingent fact that the sentence has been labelled in this particular way. As I am dealing with necessity here I could not then apply the rule of necessitation.
} 
would be very difficult: it is a complicated (non-recursive) task to decide if a sentence refers to such a predicate predicate.

It is usually assumed that the semantic paradoxes can be blocked by applying predicates such as 'is true', 'is necessary', or 'is a priori' only to sentences that do not contain the respective predicate. This is not to say that this solution is generally accepted; it is only thought to be safe even though it might be rejected by many as too restrictive. The above inconsistency, however, shows that the solution falters if it is applied to two predicates simultaneously. Thus even the highly restrictive language-level method is insufficient for blocking inconsistencies arising from the interaction of two modal predicates.

One could now impose more restrictions on the axioms and rules A1-A4: one could allow only instances of A1 where the instantiating sentences contains neither an occurrence of 'is a priori' nor an occurrence of 'is necessary'. Curtailing the axioms and rules A1-A4 in this way would block the derivation of the contradiction; but this solution comes at a high price: predicates such as 'is necessary' would no longer be applicable to sentences containing predicates like 'is priori', 'is true', 'is known', and so on. Basically one would prohibit any interaction between modal and semantic predicates and thereby declare many philosophical accounts concerning the relation between these notions as ill conceived because they do not conform with the syntactic restrictions on the axioms for these notions.

Moreover, it seems very hard to predict which axioms for different modal notions might interact in a detrimental way. It no longer seems sensible to develop theories of various modal notions separately. In order to avoid inconsistencies, when developing the theory of a modal notion, one would also have to keep an eye on all other modal notions. Basically one would have to develop the theory of all these notions with one fierce sweep, which seems next to impossible as the list of modal notions is fairly long, diverse, and perhaps even open ended.

At any rate, the problems arising from the interaction of these predicates can be resolved by eliminating modal predicates. If necessity, aprioricity, and so on are conceived as modal operators only, then diagonalisation is not possible and the interaction of the various concepts can be studied in intensional logics featuring modal operators for the different notions. 


\section{FOURTH BENEFIT: ONTOLOGY}

On the standard semantics, predicates apply to objects. Hence, if 'is necessary' and so on are predicates, they need to apply to objects. Philosophers have attributed necessity to propositions conceived as language independent objects, to sentence types or tokens of an ideal language or a language of thought, or to sentence types or tokens of a natural language. Even if one settles for propositions, there are disparate views on how propositions are individuated.

I am not generally worried about accepting objects and, in particular, abstract objects of which necessity can be predicated. I am worried about the requirement for a unified account: usually analyticity is conceived as a predicate of sentences, while necessity is conceived as a property of propositions and being known is presumably also a property of propositions but of more finely grained propositions than those propositions of which necessity is predicated. One might be tempted to say that logically equivalent sentences of a language express the same proposition as far as necessity is concerned but not as far as being known is concerned as the proposition expressed by a sentence can be known while the proposition expressed by a logically equivalent sentences is not known. Of course there is an extensive literature on the ontology of beliefs and of propositions in general, and I cannot go into the details here, but if, for instance, as it is usually done, necessity is predicated of (language independent) propositions and analyticity of predicates, then nothing that is analytic can be necessary and vice versa, simply because sentences are not propositions and vice versa.

On the operator account one needs a unified account of propositions (or sentences) that can be used for all modal and semantic predicates: analyticity must be applicable, in some way, to propositions; or necessity must be applicable to sentences. Alternatively, one could understand a claim like

There are necessary beliefs that are not analytic

(where beliefs are neutral between sentences and propositions) as shorthand for a more complicated claim of the following kind:

There are necessary propositions that are expressed by (some?) (only by?) sentences that are not analytic. 
On such an approach relations connecting the objects to which modal predicates are attributed are needed. Besides the relation used above that obtains between sentences and the propositions they express, one would presumably also invoke a relation that allows to go from finer grained propositions to coarse grained propositions and vice versa. At any rate a sizable ontological apparatus is needed to accommodate various modal predicates into a common framework.

Developing such an apparatus would have to be prior to an analysis of modal notions. However, if there is hope to reduce modal predicates to modal operators, then there is also hope to bypass the need for an intricate and comprehensive ontological theory. On the operator account, no objects to which modal predicates can be attributed are needed.

Thus not only the paradoxes arising from the interaction of modal predicates but also ontological problems impede the development of a unified framework in which various modal and semantic notions can be studied simultaneously.

\section{REDUCTIONS}

I have argued in this paper that there are good reasons for trying to eliminate modal predicates and to retain only modal operators. In the present section I'll sketch some attempts to perform this elimination. It is to be expected that the elimination comes at a certain price if quantified statements involving modal notions still are to be expressible. Here I'll not reach a conclusion, but I'll indicate some aspects that will have to be taken into account.

Above I have shown how one might go about eliminating the modal predicate from sentences like the following by using modal operators:

The proposition that water is $\mathrm{H}_{2} \mathrm{O}$ is necessary.

Only when one turns to quantified statements as (i)-(iii) above, the elimination of modal predicates cannot be carried in a straightforward way. Various proposals have been made for formalising such sentences using an operator rather than a predicate.

Universally quantified statements are often formalised as schemata. The sentence saying that all laws of physics are necessary would be expressed by the schema $L \varphi \rightarrow \square \varphi$, where $L$ is an operator expressing 'it's a 
law of physics that' and $\varphi$ ranges over all sentences of the language. One obvious problem is that now one is forced to reexpress predicates like 'is a law of physics' as operators, which might add additional problems as one might want to treat it as a predicate in 'There are no laws of physics.'

Even if this problem can be solved, this approach will work only for simple universally quantified sentences; but if the quantification is embedded into other connectives and quantifiers then they would also have to be pushed into the metalanguage, so that the formalisation of many sentences would end in an unhappy mix of expressions in the object- and the metalanguage. Thus I cannot see this strategy succeed.

In order to express this quantified statements within the objectlanguage one could invoke special quantifiers. The sentence saying that all laws of physics could then be formalised as $\forall \varphi(\operatorname{Law}(\varphi) \rightarrow \square(\varphi))$ using a predicate 'Law $(x)$ ', a modal operator $\square$ for necessity and the special quantifier with the variable $\varphi$ that stands in object and sentence places. Of course one would need a semantics for this new kind of quantifier that does not reintroduce the ontological problems of the predicate account through the backdoor: in particular, if $\forall \varphi$ is taken as a quantifier ranging over sentences, propositions or the like, it is not unlikely that one will be confronted with the same problems that were to be solved in virtue of the elimination of the modal operators. A substitutional reading of this quantifier seems to overcome these problems.

Kripke (1976) investigated this kind of substitutional quantification in close connection with his work on truth (1975). In fact one can show that languages with this kind of quantification and languages with a truth predicate are intertranslatable.

To me it seems that the use of a truth predicate is much closer to natural language (and in the end conceptually leaner than the use of special quantifiers). In order to express quantification involving modal notions we often use an adverb plus the truth predicate as in 'Some a priori beliefs aren't necessarily true' instead of 'Some a priori beliefs aren't necessary.'

The use of a truth predicate comes at a price, however. The use of the truth predicate seems to reintroduce the problems I tried to block by eliminating modal notions: truth needs to apply to objects, so there will be ontological problems; and truth is prone to paradox. But I think that there are some important gains in a reduction of modal notions to modal operators and a truth predicate, which I will highlight in the following.

First, logicians and philosophers can continue to use modal logic for analysing modal notions such as necessity. The quantification problem is 
solved via the truth predicate and no predicate of necessity is needed. Thus one will need a theory of truth, but one can retain intensional logics and possible-worlds semantics as a general framework for analysing modal notions.

Second, only the paradoxes of truth need to be solved. Paradoxes arising from self-reference do not arise for modal notions anymore as they are treated as operators that cannot be used in diagonalisations. At least all the problems about paradoxes are concentrated on truth. Montague's and similar paradoxes can be regained in the framework proposed: but they become truth-theoretic paradoxes and they do not threaten the theory of necessity and other modal notions different from truth.

Third, there cannot be paradoxes arising from the interaction of modal predicates, simply because there is only one such predicate left: truth.

Fourth, also the ontological problem of a unique category of objects to that all modal notions can be applied disappears as there are not any modal predicates left that need to apply to objects. Of course, truth still needs to apply to objects but one does not need any longer objects to which predicates of necessity, analyticity, and belief can be applied in the same way. One does not need anymore a common category of objects that can be necessary, be known, and so on.

In (2009) Philip Welch and I have tried to provide the formal framework for carrying out the proposed reduction of modal notions to modal operators plus a truth predicate. There are some formal obstacles to the proposed elimination of modal predicates, but they can be overcome under certain assumptions, which are far from being uncontroversial. For instance, in this paper we rely on Kripke's (1975) solution of the truththeoretic paradoxes. Here I do not go on to discuss further aspects of this discussion as my main topic has been only the motivation for carrying out this reduction.

In the proposed reduction the truth predicate is used for expressing generalisations. Thus, according to many philosophers, we have made use of a merely deflationist truth predicate. However, the availability of a deflationist truth predicate might allow one to eliminate modal predicates and therefore enjoy the above benefits of this reduction. Thus a mere device of generalisation might impinge on issues in ontology, the paradoxes and other central areas of philosophy. Deflationist truth might be only a tool, but, if used in the right way, it might turn out to be a sharp razor. 


\section{REFERENCES}

Halbach, Volker (2006), 'How Not to State the T-sentences', Analysis 66, pp.276-280. Correction of printing error in vol.67, p.268.

Halbach, Volker (2008), 'On a Side Effect of Solving Fitch's Paradox by Typing Knowledge', Analysis 68, pp.114-120.

Halbach, Volker, Hannes Leitgeb and Philip Welch (2003), 'Possible Worlds Semantics for Modal Notions Conceived as Predicates', Journal of Philosophical Logic 32, pp.179-223.

Halbach, Volker and Philip Welch (2009), 'Necessities and Necessary Truths: A Prolegomenon to the Metaphysics of Modality', Mind. to appear.

Horsten, Leon and Hannes Leitgeb (2001), 'No Future', Journal of Philosophical Logic 30, pp.259-265.

Kripke, Saul (1975), 'Outline of a Theory of Truth', Journal of Philosophy 72, pp.690-712. reprinted in Martin (1984).

Kripke, Saul (1976), 'Is There a Problem about Substitutional Quantification?', in G.Evans and J.McDowell, eds, Truth and Meaning: Essays in Semantics, Clarendon Press, Oxford, pp.325-419.

Martin, Robert L., ed. (1984), Recent Essays on Truth and the Liar Paradox, Clarendon Press and Oxford University Press, Oxford and New York.

Montague, Richard (1963), 'Syntactical Treatments of Modality, with Corollaries on Reflexion Principles and Fnite Axiomatizability', Acta Philosophica Fennica 16, pp.153-167. Reprinted in (Montague 1974, pp.286-302).

Montague, Richard (1974), Formal Philosophy: Selected Papers of Richard Montague, Yale University Press, New Haven and London. Edited and with an introduction by Richmond H. Thomason.

Niebergall, Karl-Georg (1991), Simultane objektsprachliche Axiomatisierung von Notwendigkeits- und Beweisbarkeitsprädikaten, Master's thesis, Ludwigs-Maximilians-Universität München.

Petzolt, Sebastian (2009), 'A Paradox Remains', Logique et Analyse. to appear. 
